\title{
Testing Gravity-Driven Collapse of the Wavefunction via Cosmogenic Neutrinos
}

\author{
Joy Christian \\ Perimeter Institute, 31 Caroline Street North, Waterloo, Ontario N2L 2Y5, Canada, and \\ Department of Physics, University of Oxford, Parks Road, Oxford OX1 3PU, England
}

\begin{abstract}
It is pointed out that the Diósi-Penrose ansatz for gravity-induced quantum state reduction can be tested by observing oscillations in the flavor ratios of neutrinos originating at cosmological distances. Since such a test would be almost free of environmental decoherence, testing the ansatz by means of a next generation neutrino detector such as IceCube would be much cleaner than by experiments proposed so far involving superpositions of macroscopic systems. The proposed microscopic test would also examine the universality of superposition principle at unprecedented cosmological scales.
\end{abstract}

PACS numbers: 03.65.Ta, 14.60.Pq, 95.85.Ry

In the 1950s Feynman observed that a possible gravityinduced failure of quantum mechanics for objects as small as the Planck mass $\left(\sim 10^{-5}\right.$ grams $)$ is not inconsistent with the existing physical evidence [1]. Remarkably, this elementary observation remains unchallenged even today, when we have gained almost unshakable confidence in the universality of quantum mechanics. Compounded by persistent conceptual problems of both quantum theory and quantum gravity 2], time and again this fact has inspired suggestions of a gravity-driven reduction of the state vector [3] [4] [5], albeit in a considerably evolved form than its preliminary inception by Feynman [1].

Noteworthy among these are the specific proposals put forward by Diósi [4] and Penrose [5], who independently arrive at a phenomenological ansatz for the time scale beyond which quantum superpositions may become unstable. This "duration of quantal stability" turns out to be experimentally testable, and can be expressed in a form resembling the mean life of an unstable particle:

$$
\mathcal{T} \sim \frac{\hbar}{\Delta E_{G}}
$$

where $\Delta E_{G}$ is the gravity-induced ill-definedness in the energy of a given system in superposition of two states.

There have been several experimental proposals to test this ansatz [5] 6] [7]. Remarkably, one of these proposals [7] purports to superpose a mirror of some $10^{-10}$ grams, which is only about 5 orders of magnitude short of the Planck mass. These proposals are, of course, only a small part of the ongoing drive to experimentally push the boundaries of superposition principle as far up the macroscopic scale as possible [8]. All of these efforts are hampered, however, by one major difficulty. Due to the intractability of environmentally induced decoherence for such large systems, it is usually extremely difficult to distinguish any genuine state reduction scheme from the effective decoherence resulting from a subjective omission of the environmental degrees of freedom 9 .

There is, however, nothing in ansatz (11) that necessitates a macroscopic system for its validity. Indeed, if the ansatz was meant only for such large systems, it would not bear the fundamental and universal significance attached to it by Diósi and Penrose. Put differently, even if the ansatz is verified for macroscopic systems, it cannot be accepted as a truly fundamental feature of the world until it is also verified for elementary systems. Therefore, here we propose to test the ansatz on neutrinos, originating at cosmological distances. Since neutrinos are electromagnetically neutral and sensitive only to the weak and gravitational interactions, the chances of them decohering within the cosmic vacuum are negligible. As a result, cosmogenic neutrinos form an ideal system for testing any scheme of gravity-driven state reduction.

To appreciate this, in what follows we first take a closer look at the rational behind ansatz (10), then review the theory of neutrino flavor oscillations, and, finally, extract deviations from the quantum mechanically expected flavor ratios by applying the ansatz to massive neutrinos.

The physics of Diósi-Penrose ansatz.-Since Penrose's proposal is minimalist in conception (i.e., it relies only on the first principles of quantum mechanics and general relativity), we shall follow his reasoning $[\underline{5}$. He considers two quantum states of a given mass, $\left|\Psi_{1}\right\rangle$ and $\left|\Psi_{2}\right\rangle$, each stationary on its own, and possessing the same energy $E$ :

$$
i \frac{\partial}{\partial t}\left|\Psi_{1}\right\rangle=E\left|\Psi_{1}\right\rangle, \quad i \frac{\partial}{\partial t}\left|\Psi_{2}\right\rangle=E\left|\Psi_{2}\right\rangle
$$

(henceforth we mostly use Planck units: $\hbar=c=G=1$ ). In standard quantum mechanics linearity necessitates that a possible superposition of these two states, such as $|\mathcal{X}\rangle=\lambda_{1}\left|\Psi_{1}\right\rangle+\lambda_{2}\left|\Psi_{2}\right\rangle$, must itself be a stationary state with the same energy. However, when gravitational fields of the two masses are taken into account, in general each of the original states would correspond to two entirely different spacetimes. The principles of general relativity would then dictate that the time-translation operators ' $\frac{\partial}{\partial t}$ ', corresponding to the action of the timelike Killing vector fields of the two (stationary) spacetimes, would also be quite distinct form one another. On the other hand, when these two Killing fields happen not to be too different, there would be only a slight ill-definedness in the action of $\frac{\partial}{\partial t}$, and that would be reflected in the 
energy of the system. Penrose uses this gravity-induced "error" in energy, $\Delta E_{G}$, as an approximate measure of instability of the superposition, and postulates the mean lifetime $\mathcal{T}$ of such a state to be $\left[\Delta E_{G}\right]^{-1}$, as in (1), with two decay modes being the component states $\left|\Psi_{1}\right\rangle$ and $\left|\Psi_{2}\right\rangle$ with probabilities $\left|\lambda_{1}\right|^{2}$ and $\left|\lambda_{2}\right|^{2}$, respectively.

Penrose next suggests that this measure of instability, $\Delta E_{G}$, can be estimated in terms of the incompatibility between the notions of free fall within the two spacetimes. At some identifiable event, let $\mathbf{a}_{1}$ and $\mathbf{a}_{2}$ be the acceleration 3 -vectors of the free-fall motions in the two respective spacetimes. Then $\Delta E_{G}$ can be estimated as

$$
\Delta E_{G} \approx \xi \int_{\Sigma_{t}}\left(\mathbf{a}_{1}-\mathbf{a}_{2}\right) \cdot\left(\mathbf{a}_{1}-\mathbf{a}_{2}\right) d \mathbf{r},
$$

where the integrand is a coordinate-independent scalar quantity, $\Sigma_{t}$ represents a three-dimensional hypersurface at an instant of time $t$, and $\xi \geq 0$ is an arbitrary dimensionless parameter (in what follows, this parameter will provide a phenomenological handle on the "strength" of quantum state reduction). Of course, in Newtonian approximation $\mathbf{a}_{1}$ and $\mathbf{a}_{2}$ are simply the forces per unit test mass: $\mathbf{a}_{1}=-\nabla \Phi_{1}$ and $\mathbf{a}_{2}=-\nabla \Phi_{2}$, where $\Phi_{1}$ and $\Phi_{2}$ are the respective gravitational potentials for the two spacetimes. Therefore, using the Poisson's equation $\nabla^{2} \Phi(\mathbf{r})=4 \pi \rho(\mathbf{r})$, the estimate (3) can be reduced to

$$
\Delta E_{G} \approx 4 \pi \xi \iint \frac{\left[\rho_{1}(\mathbf{r})-\rho_{2}(\mathbf{r})\right]\left[\rho_{1}\left(\mathbf{r}^{\prime}\right)-\rho_{2}\left(\mathbf{r}^{\prime}\right)\right]}{\left|\mathbf{r}-\mathbf{r}^{\prime}\right|} d \mathbf{r} d \mathbf{r}^{\prime},
$$

where $\rho_{1}$ and $\rho_{2}$ are the two respective mass distributions responsible for the two spacetimes [5]. It is worth noting here that this ill-definedness in energy is based on the gravitational energy of the system itself, and not on the energy of any externally present fields, although the latter may play an indirect role in some cases. In fact, it is essentially the gravitational self-energy of the difference between the two superposed mass distributions.

The order of magnitude for the mean life $\mathcal{T}$ based on the expression (4) can now be estimated to be simply $\Delta r / m^{2}$, where $m$ is the rest mass of the system, and $\Delta r$ is the spread in the position of the system between its two superposed states. For example, in the case of a nucleon, with $\Delta r$ taken to be its strong interaction range, the mean life of a superposition of its states turns out to be over $10^{7}$ years; whereas for systems as large as a speck of dust of mass $10^{-4}$ grams and position spread $1 \mathrm{~mm}$, it plunges to some $10^{-13}$ seconds (cf. [4] [5]). Evidently, the postulated mean life $\mathcal{T}$ reproduces the phenomenology of quantum state reduction quite compellingly.

Despite this predicted astronomically long mean life of superpositions for elementary particles, the proposed ansatz turns out to be testable for cosmogenic neutrinos.

Theory of neutrino oscillations. - The remarkable phenomena of neutrino oscillations are due to the fact that neutrinos of definite flavor states $\left|\nu_{\alpha}\right\rangle, \alpha=e, \mu$, or $\tau$, are not particles of definite mass states $\left|\nu_{j}\right\rangle, j=1,2$, or 3 , but are superpositions of the definite mass states [10]:

$$
\left|\nu_{\alpha}\right\rangle=\sum_{j} U_{\alpha j}^{*}\left|\nu_{j}\right\rangle
$$

with $U$ being the (time-independent) leptonic mixing matrix. By the same token, neutrinos of definite mass states are superpositions of the definite flavor states: $\left|\nu_{j}\right\rangle=\sum_{\beta} U_{\beta j}\left|\nu_{\beta}\right\rangle$, with the mixing matrix being subject to the unitarity constraint $\sum_{j} U_{\alpha j}^{*} U_{\beta j}=\delta_{\alpha \beta}$. As a neutrino of definite flavor state propagates through vacuum for a long enough laboratory time, the heavier mass-eigenstates in (515) lag behind the lighter ones, and the neutrino transforms itself into a different flavor state. The probability for this transition from one flavor state to another can be easily obtained as follows. In the rest frame of each $\left|\nu_{j}\right\rangle$, where the proper time is $\tau_{j}$, plane wave analysis leads to the Schrödinger equation

$$
i \frac{\partial}{\partial \tau_{j}}\left|\nu_{j}\left(\tau_{j}\right)\right\rangle=m_{j}\left|\nu_{j}\left(\tau_{j}\right)\right\rangle
$$

with a solution $\left|\nu_{j}\left(\tau_{j}\right)\right\rangle=e^{-i m_{j} \tau_{j}}\left|\nu_{j}(0)\right\rangle$, where $m_{j}$ is the eigenvalue of the mass-eigenstate $\left|\nu_{j}(0)\right\rangle$. In terms of the coordinate time $t$ and position $\mathbf{r}$ in the laboratory frame, this phase factor takes the familiar form

$$
e^{-i\left(E_{j} t-\mathbf{p}_{j} \cdot \mathbf{r}\right)},
$$

where $E_{j}$ and $\mathbf{p}_{j}$ are, respectively, the energy and momentum associated with the definite mass state $\left|\nu_{j}(0)\right\rangle$.

Now neutrinos are highly relativistic particles, which permits the assumption that $t \approx|\mathbf{r}|=L$, where $L$ is the distance traveled by them before detection. Moreover, assuming that they are produced with the same energy $E$ regardless of which state $\left|\nu_{j}(0)\right\rangle$ they are in (and that $m_{j} \ll E$ ), up to the second order in $m_{j}$ the dispersion relation gives the following expression for their momenta,

$$
p_{j}=\sqrt{E^{2}-m_{j}^{2}} \approx E-\frac{m_{j}^{2}}{2 E},
$$

which, along with the assumption $t \approx L$, reduces the phase factor in (7) to $e^{-i\left(m_{j}^{2} / 2 E\right) L}$. Consequently, in the laboratory frame, and up to the second order in $m_{j}$, the time evolution of the neutrino flavor state (5) is given by

$$
\left|\nu_{\alpha}(t)\right\rangle=\sum_{\beta} \sum_{j} U_{\alpha j}^{*} e^{-i\left(m_{j}^{2} / 2 E\right) L} U_{\beta j}\left|\nu_{\beta}(0)\right\rangle .
$$

As a result, the transition probability for the neutrinos to "oscillate" from a given flavor state, say $\left|\nu_{\alpha}(0)\right\rangle$, to another flavor state, say $\left|\nu_{\beta}(t)\right\rangle$, is given by

$$
\begin{aligned}
P_{\alpha \beta}(E, L) & :=P_{\nu_{\alpha} \rightarrow \nu_{\beta}}(E, L)=\left|\left\langle\nu_{\beta}(0) \mid \nu_{\alpha}(t)\right\rangle\right|^{2} \\
= & \delta_{\alpha \beta}-\sum_{j \neq k} U_{\alpha j}^{*} U_{\alpha k} U_{\beta j} U_{\beta k}^{*}\left[1-e^{-i\left(\Delta m_{j k}^{2} / 2 E\right) L}\right],
\end{aligned}
$$


where $\Delta m_{j k}^{2} \equiv m_{k}^{2}-m_{j}^{2}>0$ is the difference in the squares of the two masses. From this transition probability it is clear that the experimental observability of neutrino oscillations is determined by the quantum phase

$$
\Phi:=2 \pi \frac{L}{L_{O}},
$$

where $L_{O}(E, m):=4 \pi E / \Delta m_{j k}^{2}$ is the energy-dependent oscillation length. Thus, flavor changes would be observable whenever the propagation distance $L$ is of the order of the oscillation length $L_{O}$. Therefore, in what follows it would suffice to concentrate on these two variables.

Applying Diósi-Penrose ansatz to massive neutrinos.It is evident from ansatz (1) that the proposed mean life of superpositions is independent of the speed of light, and hence applicable to both nonrelativistic as well as relativistic systems, including ultra-relativistic neutrinos. Moreover, for our purposes it would not be incongruous to estimate the spacetime distortions due to neutrinos themselves by treating them as classical spinning particles. Accordingly, let us consider a spherically symmetric gravitating body of mass $m$ and angular momentum $\mathbf{s}$. If the gravitational field produced by the body is sufficiently weak, then, in an approximate global inertial frame, it can be described by the following well-known solution of the linearized Einstein's field equations:

$$
\begin{aligned}
d s^{2} \approx & -\left(1-\frac{2 m}{r}\right) d t^{2}-\frac{4|\mathbf{s}|}{r} \sin ^{2} \theta d t d \phi \\
& +\left(1+\frac{2 m}{r}\right)\left(d r^{2}+r^{2} d \theta^{2}+r^{2} \sin ^{2} \theta d \phi^{2}\right) .
\end{aligned}
$$

This is essentially a Newtonian line element, apart from the off-diagonal term involving the magnitude $|\mathbf{s}|$ of the intrinsic angular momentum of the body. Now, in the case of a neutrino the magnitude of $\mathbf{s}$ is simply $\frac{\hbar}{2}$, which, in ordinary units, is some 16 orders of magnitude per second smaller than the estimated (active) neutrino mass (measured to be $<2.3 \mathrm{eV}[11]$ ). Therefore, it would be adequate for our purposes to consider only the Newtonian part of the gravitational field due to the neutrino mass, and neglect the off-diagonal contribution due to its spin.

As an excellent approximation, it is then possible to apply Penrose's Newtonian prescription (44) to each of the three pairs of neutrino states in the superposition (5). Moreover, provided we continue to take the expectation value for the neutrino mass-distribution to be a uniform sphere of effective radius $a_{j}$, this two-body Newtonian prescription can be easily calculated to be

$$
\Delta E_{G}^{j, k} \approx 8 \pi \xi\left[\frac{3 m_{j}^{2}}{5 a_{j}}+\frac{3 m_{k}^{2}}{5 a_{k}}-\frac{m_{j} m_{k}}{\left|\mathbf{r}_{j}-\mathbf{r}_{k}\right|}\right],
$$

with $\left|\mathbf{r}_{j}-\mathbf{r}_{k}\right|$ being the displacement between the two superposed mass-eigenstates resulting from their journey.
The particular shape of the smearing introduced here to avoid the self-energy divergence has little effect on what follows 12]. More importantly, it is manifest from (13) that the product $\Delta E_{G}^{j, k} \times d L$ is Lorentz invariant. Now for ultra-relativistic neutrinos the usual spreading of the wavepacket can be easily shown to be negligible [13], but within a neutrino beam of definite energy the different mass-eigenstates $\left|\nu_{j}\right\rangle$ in the superposition (5) travel at slightly different speeds $\beta_{j}$, producing the displacement

$$
\left|\mathbf{r}_{j}-\mathbf{r}_{k}\right|=\left(\beta_{j}-\beta_{k}\right) t \approx \frac{\left(p_{j}-p_{k}\right)}{E} L \approx \frac{\Delta m_{j k}^{2}}{2 E^{2}} L .
$$

Here the last relation follows from (8), and we have used the relativistic identity $\beta=p / E$, as well as continued to assume $t \approx L$ and taken the $k^{t h}$ neutrino to be the heavier (and hence the slower) of the two partners. Using (14), the measure (13) can now be rewritten in terms of neutrino parameters - such as energy, propagation length, and the mass-squared difference - as follows:

$$
\Delta E_{G}^{j, k}(L) \approx 8 \pi \xi\left[\frac{3\left(m_{j}+m_{k}\right)}{5 G_{F}}-\frac{2 m_{j} m_{k} E^{2}}{\Delta m_{j k}^{2} L}\right],
$$

where we have taken the effective radii $a_{j}$ to be $\approx G_{F} m_{j}$, with $G_{F}$ being the Fermi constant of weak interactions.

The effect of this gravity-induced ill-definedness on the off-diagonal matrix elements of the statistical operator corresponding to the superposition (9) can now be easily worked out (cf. [12]). Unsurprisingly, it turns out to be a time-dependent modification of the matrix elements,

$$
U_{\alpha j}^{*} U_{\alpha k} U_{\beta j} U_{\beta k}^{*} \longrightarrow e^{-\left[\int_{D}^{L} \Delta E_{G}^{j, k}\left(L^{\prime}\right) d L^{\prime}\right]} U_{\alpha j}^{*} U_{\alpha k} U_{\beta j} U_{\beta k}^{*},
$$

where the integrand-with definition $\Delta E_{G}^{j, k}(D) \equiv 0$-is the "decay constant" corresponding to the "mean life" $\mathcal{T}$ in (11). As a result of this non-unitary modification, the transition probability (10) for flavor oscillations would acquire a time-dependent "damping factor":

$$
\begin{aligned}
P_{\alpha \beta}(E, L) \longrightarrow & \delta_{\alpha \beta}-\sum_{j \neq k} U_{\alpha j}^{*} U_{\alpha k} U_{\beta j} U_{\beta k}^{*} \\
& \times\left[1-e^{-i\left(\Delta m_{j k}^{2} / 2 E\right) L-\int_{D}^{L} \Delta E_{G}^{j, k}\left(L^{\prime}\right) d L^{\prime}}\right] .
\end{aligned}
$$

Now, in the absence of the damping factor (i.e., within a unitary mechanics), it is clear from the phase (11) that flavor changes can be observable only when the propagation distance $L$ of neutrinos is about the same size as their oscillation length $L_{O}$-i.e., only when the condition

$$
\Delta m_{j k}^{2} \sim \frac{4 \pi E}{L}
$$

is satisfied. Substituting this observability condition into the evaluation of the equation $e^{-\left[\int_{D}^{L} \Delta E_{G}^{j, k}\left(L^{\prime}\right) d L^{\prime}\right]}=e^{-1}$ 
then yields the following condition for observability of the proposed instability in quantum superpositions:

$L \sim \frac{l_{P}}{8 \pi \xi}\left[\frac{3\left(m_{j}+m_{k}\right)}{5 m_{P}^{3} G_{F}}-\frac{m_{j} m_{k} E}{2 \pi m_{P}^{3}} \ln \left\{\frac{6 \pi e\left(m_{j}+m_{k}\right)}{5 G_{F} m_{j} m_{k} E}\right\}\right]^{-1}$

where, for convenience, we have explicated the units by means of Planck length $\left(l_{P}\right)$ and Planck mass $\left(m_{P}\right)$.

From this observability condition it is easy to work out that - assuming the values of masses $m_{j} \approx m_{k} \approx 2 \mathrm{eV}$ 11] - the Diósi-Penrose scheme for state reduction can be either ruled out or verified for the values of $E$ and $L$ in the (approximate) ranges of $[0,2.3] \times 10^{23} \mathrm{eV}$ and $[0.7,15] \times 10^{9}$ light-years, respectively. As a result, provided cosmogenic neutrinos are at our disposal, an upper bound of order $10^{-2}$ can be comfortably placed on the free parameter $\xi$. In fact, it may even be possible to place an upper bound as strong as of order $10^{-3}$ on this parameter. This is clear from the nature of the transition probability (17) itself, which would change significantly (thereby altering the observable flavor ratios from the quantum mechanical expectations) even when the nonunitary Diósi-Penrose damping is as weak as $e^{-0.1}$.

Observability of the non-unitary flavor oscillations.Ultra-high-energy neutrinos from cosmologically distant sources such as active galactic nuclei (AGN) and gamma ray bursters (GRBs) are generally believed to be produced as secondaries of cosmic ray protons interacting with ambient matter and photon fields [14]. Such protonproton and proton-photon interactions produce neutral and charged pions, which, in turn, decay into neutrinos via the chain: $\pi^{+} \rightarrow \mu^{+} \nu_{\mu} \rightarrow e^{+} \nu_{e} \bar{\nu}_{\mu} \nu_{\mu}$. From the very inception, these interactions have been thought to provide a "guaranteed" source of cosmogenic neutrinos. Moreover, although the absolute flux of the different flavor states of such neutrinos is presently unknown, the above decay chain strongly suggests their relative flux ratios $\phi_{\nu_{e}}^{S}: \phi_{\nu_{\mu}}^{S}: \phi_{\nu_{\tau}}^{S}$ at the source to be $\frac{1}{3}: \frac{2}{3}: \frac{0}{3}$.

Now, neutrinos - being stable and neutral particlespoint back to their sources, thereby providing vital information about their propagation lengths $L$, which can then be further consolidated by the coincident data on the cosmological redshifts of the sources [15]. Furthermore, being only weakly interacting, in the absence of the Diósi-Penrose decay [provided condition (18) is satisfied] their flavor states (9) would maintain quantum coherence while propagating through the cosmic vacuum. Given the above initial flux ratios of neutrino flavors, this coherence would then be reflected in the flavor fluxes observed at a terrestrial detector, which can be easily calculated as

$$
\phi_{\nu_{\beta}}^{D}(E, L)=\sum_{\alpha=e, \mu, \tau} P_{\alpha \beta}(E, L) \phi_{\nu_{\alpha}}^{S},
$$

where the transition probabilities $P_{\alpha \beta}$ are given by (10). The corresponding flux ratios $\phi_{\nu_{e}}^{D}: \phi_{\nu_{\mu}}^{D}: \phi_{\nu_{\tau}}^{D}$ observed at a detector can thus be compared with those predicted via the non-unitary transition probabilities (17), provided the mass-dependent observability relations (19) between the variables $E$ and $L$ in (20) are satisfied, and sizable fluxes of relevant flavors are collected at Earth.

Fortunately, recent estimates of cosmogenic neutrino fluxes suggest that this is indeed feasible. For example, the authors of Ref. 16] estimate sizable fluxes of neutrinos from cosmologically distant sources in the energy ranges up to and beyond the threshold of $10^{21} \mathrm{eV}$. In fact, a few neutrinos in the $\mathrm{MeV}$ range from a distant supernova have already been observed 17. What is more, there are a large variety of neutrino detectors under construction at present, or planned to be operational in the near future, designed to be sensitive to a wide range of neutrino energies [16] 18] 19. Their ability to measure the flavor ratios of cosmogenic neutrinos at high precision has also been demonstrated in Ref. [20]. Therefore, a test of the Diósi-Penrose ansatz by means of observing flavor ratios of cosmogenic neutrinos appears to be quite feasible.

I am grateful to Roger Penrose for discussions on his ideas about gravity-induced state reduction, and to Lajos Diósi for his constructive comments on the manuscript.

[1] R. Feynman, in "Feynman Lectures on Gravitation", ed. B. Hatfield (Addison-Wesley, Reading, MA, 1995).

[2] J. Christian, in "Physics Meets Philosophy at the Planck Scale", eds. C. Callender and N. Huggett (Cambridge University Press, Cambridge, 2001).

[3] F. Károlyházy, Il Nuovo Cimento. 42 A, 390 (1966); I. C. Percival, Proc. Roy. Soc. Lond. A 451, 503 (1995); P. Pearle and E. Squires, Found. Phys. 26, 291 (1996);

[4] L. Diósi, Phys. Lett. 105 A, 199 (1984); Phys. Lett. A 120, 377 (1987); Phys. Rev. A 40, 1165 (1989).

[5] R. Penrose, Gen. Relativ. Gravit. 28, 581 (1996); Phil. Trans. R. Soc. Lond. A 356, 1927 (1998); "The Road to Reality" (Jonathan Cape Publishers, London, 2004).

[6] P. Pearle and E. Squires, Phys. Rev. Lett. 73, 1 (1994); S. Bose et al., Phys. Rev. A 59, 3204 (1999); C. Henkel et al., Phys. Rev. A 70, 023810 (2004).

[7] W. Marshall et al., Phys. Rev. Lett. 91, 130401 (2003).

[8] M. Arndt et al., Nature (London) 401, 680 (1999).

[9] E. Joos and H.D. Zeh, Z. Phys. B59, 223 (1985).

[10] B. Kayser, hep-ph/0104147 M. Beuthe, Physics Reports 375, 105 (2003).

[11] Ch. Kraus et al., hep-ex/0412056

[12] G. C. Ghirardi, R. Grassi, and A. Rimini, Phys. Rev. A 42, 1057 (1990).

[13] A. Bottino et al., Mod. Phys. Lett. A 4, 1799 (1989).

[14] H. Athar, hep-ph/0212387 and hep-ph/0308188

[15] C. Lunardini and A. Y. Smirnov, Phys. Rev. D 64, 073006 (2001).

[16] O.E. Kalashev et al., Phys. Rev. D 66, 063004 (2002).

[17] R. M. Bionta et al., Phys. Rev. Lett. 58, 1494 (1987).

[18] C. Spiering, Nucl. Phys. (Proc. Suppl.) 125, 1 (2003).

[19] J. Christian, Phys. Rev. D 71, 024012 (2005).

[20] J.F. Beacom et al., Phys. Rev. D 68, 093005 (2003). 